\title{
Reflective grating interferometer: a versatile and simple system for education in optics
}

Sergio De Nicola, Pietro Ferraro, Andrea Finizio, Giovanni Pierattini

Sergio De Nicola, Pietro Ferraro, Andrea Finizio, Giovanni Pierattini, "Reflective grating interferometer: a versatile and simple system for education in optics," Proc. SPIE 3190, Fifth International Topical Meeting on Education and Training in Optics, (8 December 1997); doi: 10.1117/12.294371

Event: Fifth International Topical Meeting on Education and Training in Optics, 1997, Delft, Netherlands 
Reflective grating interferometer: a versatile and simple system for education in optics

S. De Nicola, P.Ferraro*, A.Finizio and G.Pierattini

Istituto di Cibernetica del CNR, via Toiano 6 - 80072 Arco Felice - Italy

* Also with Istituto Professionale di Stato per l'Industria e l'Artigianato "G.L.Bernini", Via Arco Mirelli 19/A, 80122

Napoli

\begin{abstract}
Recently has been developed a two component interferometer that has several advantages when used for education and training in optics. It is a wavefront division interferometer based on the use of a reflective grating that is used for recombination of two portion of one plane wavefront, obtained using a low cost parabolic mirror of commercial telescopes. The alignment operation is performed setting the mirror and the reflective grating at 90 degree each other. Interference fringe pattern are present in all the volume in front the reflective grating interferometer and a screen or a CCD camera without lens can be used to visualise fringes. The interferometer being made of only two components is also very stable. Fringe pattern spacing and orientation can be simply changed rotating the mirror on a vertical axis or tilting it respectively. The fact that mirror and the grating are adjacent has some advantages on the pedagogical side because students can understand better how relative movements of the two components affect the changes in the fringe pattern. This interferometer is used not only to study interferometry but can be used also to study geometrical optics using the fringe pattern as a grid to perform Ronchi test. In such a way it is possible to conduct investigation on aberrations of optical components (spherical, astigmatism and coma) or determine focal lengths lenses by using moiré effect. In conclusion the RGI is a system that comprises, at same time, in its operation different areas of the optics: interferometry, diffraction, wavefront picture of light, geometrical optics, testing. Examples of application for education purposes are described.
\end{abstract}

Keywords: interferometry, reflective grating, focal length, Ronchi tests, aberration.

\title{
1. INTRODUCTION
}

We propose in this paper a new type of interferometer namde Reflective Grating Interferometer (RGI) that can be useful in teaching optics at all levels. The interferometer employs only two optical elements, a mirror and a reflective-type diffraction grating. With this interferometer can be conducted experimental demonstration and training in optics but with some advantges in respect to other interferometers 1 . Numerous applications and experimental demonstrations are suggested. It is worthwhile recalling that such an interferometric system is widely used in the moiré interferometric technique 2 .

\section{PRINCIPLE OF OPERATION OF THE RGI}

The basic configuration of the RGI is shown in Fig.1. An He-Ne laser beam is expanded and collimated. The beam is then reflected downward onto the interferometer. The interferometer consists of a 1200 lines $/ \mathrm{mm}$ reflective diffraction grating G and a mirror $M$, which is adjustable in tilt and mounted on a rotating base. The direction of the rulings of the grating is perpendicular to the drawing plane. The interferometer can be considered as a wavefront division interferometer. We can assume that a single plane wavefront is divided in two parts by the two optical elements. Part $A$ of the wavefront strikes the grating directly; part $B$ of the wavefront impinges the grating $G$ via mirror $M$. The angle of incidence of the wavefront is chosen so that the +1 and -1 diffraction orders from these two parts of the incident light beam are collinear along the grating normal ( $z$ axis). When the mirror $M$ is tilted slightly with respect to the grating normal, an interference pattern results from the superposition of the two diffracted orders which is observed via a CCD camera or directly on a screen. The interference pattern is a set of parallel straight fringes with a spatial frequency corresponding to $\delta=\sin \alpha / \lambda$ where $\lambda=632 \mathrm{~nm}$ is the laser wavelength and $\alpha$ is the angle between the two interfering diffraction orders. Moreover if the mirror is tilted the interference fringes can be inclined. A contrast of the visible interference pattern up to $100 \%$ is achievable. The RGI has some advantages. An advantage of this interferometer is that the object (part A), and the reference beam (part B) are subject to the same phase change if the grating is not accurately flat. This proves that the flatness of the grating surface is less important as in the common Fizeau interferometers with flat surfaces. The experimental set-up is very easy to align because we simply need to superimpose the +1 and -1 light diffraction orders at observation plane. That condition is obtained when the grating and the mirror are perpendicular to each other. Motivation for adopting this system are the simplicity in alignment, compactness, stability against vibration and reduced number of optical components which provide the necessary flexibility for various applications in the widely spread field of optical component characterisation and training in optics. Infact the mounting and alignment operations of a RGI configuration allow to involve students with almost all fundamental 
concepts and phenomena of optics: beam expansion, collimation, diffraction, diffraction gratings and interference. Furthermore trough the experiments proposed, it is possible to afford in an easy way: wavefront geometry, index of refraction of solid and liquids, measuring focal length of lenses, aberrations and moiré effect.

\section{APPLICATIONS}

In this section will be described a number of applications of the RGI in various field of optics. Each experience will be described briefly. Some of that experience has been conducted in classroom of undergraduate students (14 to 19 years old) in a public school for opticians in Italy.

\subsection{Wavefront geometry}

The RGI can be used to study the interference of various wavefront geometry with additional advantages over other interferometric configuration 1 . With the basic configuration, as said in the section 2 ., can be easily obtained parallel fringe pattern by rotating the mirror; fringes can be inclined tilting the mirror. The fringes can be seen as produced by two plane and tilted wavefronts. Apart frorm the simplicity of alignment operation, the mirror and the grating are very close each other; this helps students in understanding what happens when they move the mirror. By using more complex configurations, such as Michelson or Mach-Zehnder, students need to move one mirror or a beam splitter in respect to another mirror that is in another area of the optical bench. Students can be loose the connection between what they are moving and its effect on the fringe pattern. It is especially true when unexapanded laser beams are used; also a very small movement of one component can cause misalignment of the system. The alternate solution could be the use of a TymannGreen configuration that needs more and much expensive components and is much sensitive to external vibrations.

To show interference of a spherical wave with a plane wave is suffice to put a lens into the path of one part, say part B, of the collimated wavefront. The part A, after has been diffracted by the grating, remains plane while the wavefront of part B become spherical. The interference of a plane wave and a spherical wave creates a pattern of concentric rings, which equation is well known. By the same procedure, using a cylindrical lens, the interference between a cylindrical and a plane wave will result.

\subsection{Measuring index of refraction of solids}

For determining the refractive index $n$ of a transparent sample material of thickness $l$, we introduce it into the part A of the collimated beam. The sample has to be mounted on a rotation stage. By rotating the sample, since the thickness is constant, the part of wavefront transmitted through the sample is plane and with unchanged inclination. It will be laterally displaced. Since the optical path difference (OPD) with respect to the reference wavefront (part B) is constant over all the wavefront, the students will observe an unchanged fringe pattern geometry; fringes will shift of an amount that depends from the angle of rotation. Counting the number of fringes $N$ that cross the field of view and measuring the incidence angle $\theta$ is possible to estimate the refractive index $n .3$. In Fig. 1 , is shown the experimental set-up.

\subsection{Measuring index of refraction of liquids}

Of particular interest can be the measurement of the index of refraction of liquids using the RGI5. Infact one problem in measuring the index of refraction of liquids is that in the measurement process is affected by the presence of the cell containing the liquid. This can limit the possibility to perform laboratory activity in a classroom. In Fig. 2 is reported an alternate experimental configuration of RGI that allows to perform experiments also with liquids. The reference and the object beams (namely parts A and B) of the interferometer pass through the same cell. The cell is divided into two portions: one is filled with sample liquid while the other can be filled with a reference liquid or simply with air. This allows the measurement of differences in the sample refractive index with respect to the reference, without taking into account for the presence of the cell. It is easy to see that the paths through the walls of the cell are exactly compensated and that the OPD between the two parts A and B is caused only by the different path in the liquid (part B) and in air (part A). The measuring procedure is the same of that adopted for solids (section 3.2).

\subsection{Measuring focal length of a lens}

Modern methods to measure focal length of lenses are based on interferometry or diffraction, such as moire deflectometry (MD) and Talbot interferometry (TI). In MD and TI methods, the focal length of a lens is obtained measuring the inclination or the number of fringes produced by a moire effect between two Ronchi gratings. Recently an improvement of moiré deflectometry has been developed 5 that is based on application of the RGI and in which an interferometric fringe pattern is used instead of Ronchi gratings. The principle of operation of that technique is simply. The proposed activity can be used for experimental session in classroom to teach optics for undergraduate and graduate levels. The optical set-up used 
is shown in Fig. 3 and is the same as in ref. 6 . The pitch of the imaged fringe pattern is approximately constant, in absence of aberration, and it can be measured by counting the number of fringes that fall within a fixed aperture. The focal length of a lens can be obtained, in principle, using only the knowledge of the magnification experienced by an imaged grating. Infact, such methods as MD and TI, while based on moiré effects, have been developed to measure the magnification directly. In those moiré methods one Ronchi grating suffers a change in magnification when it is imaged by the lens and a second grating is used to display the magnification change 7,8 . When two gratings with different pitches are superimposed, a moiré effect occurs in the form of a spatial beating at the difference of their two spatial frequencies. In using either TI or $\mathrm{MD}$ is the phenomena of self-imaging occurs. The presence of the self imaging degrades the image of the projected grating, facing the students with a more complex effect, (in this case a side problem) that can cause confusion and inattention from the main objective of the experiment. The principle of the technique is the following. Considering the tested lens is set at distance $\Delta$ from the plane of the CCD array and assuming that $\Delta<f$, the pitch of the interference pattern imaged by the lens is given by

$$
p^{\prime}=p \frac{f-\Delta}{f}
$$

where $p$ is the pitch of the interference fringe pattern without the lens, and $p^{\prime}$ is the pitch of the interference fringe pattern when imaged by the tested lens at distance $\Delta$ from the center from the lens. By using eqs. (1), and by taking into account that $p=a / N$ and $p^{\prime}=a / N^{\prime}$ where $N$ and $N^{\prime}$ are the number of fringes of the interference pattern respectively with the lens and without the lens in the optical set-up and $a$ is the aperture size of the CCD array, we easily obtain the simple expression

$$
f=\Delta\left(\frac{N^{\prime}}{N^{\prime}-N}\right)
$$

It may be difficult to measure the absolute distance $\Delta$ because this distance has to be measured from the principal plane of the lens, which could be inaccessible for thicker lenses or unknown. To avoid this we write eq.(2) in different form, namely

$$
f=\frac{\varepsilon}{N} \frac{N_{1} N_{2}}{N_{2}-N_{1}}
$$

Hence, from eq.(3) we see that to determine the focal length it is necessary to measure three parameters, i.e., $N$ the number of fringes of the interferometric pattern without the lens, $N_{1}$ and $N_{2}$ the number of fringes that are inside the chosen aperture (if a CCD array is used $a=512$ pixels) measured when the lens is at distance $\Delta$ and $\Delta+\varepsilon$ respectively. Counting of the number of fringes can be performed with different methods.

\subsection{Aberration}

The RGI can also be used to evaluate aberration on optical systems with two separate configurations: testing aberrations of the present in the wave front before the mirror and the reflective grating; testing the aberration introduced by an optical components after the fringe pattern has taken place. In the first case the interferometer is a sort of a reversing and folded interferometer 9 .

\subsubsection{Coma}

In the second case it can be regarded as an angular shearing interferometer. In the experimental set-up of Fig.3, we can consider two plane waves passing through the lens. One wavefront is tilted in respect to the other. The lens introduces same aberrations on the two wavefronts. Following eq.2.36 in ref. 910 about primary aberrations developed by Kingslake, we can readily show that there is sensitivity only to comatic aberration. In Fig. $4 \mathrm{~d}$ is illustrated as appears the fringe pattern in presence of lens. What is interesting is the possibility, in this configuration, to increase sensitivity to the aberration. Infact increasing the number of fringes by rotating the mirror $M$ it is possible to set the pitch of the interference pattern to comparable to the size (10 microns) of the pixel of the CCD array. Spatial beating will occur between the fringe pattern and the pixels of the CCD array. In Fig. 4a,b,c are shown as appear the beating pattern due to presence of aberration, for three different positions of the lens. 


\subsubsection{Astigmatism}

If a cylinrical lens is used instead of a spherical, and if the finges are inclined in respect to the axis of the cylinder moving, then the "capriole" 11 will be observed showing the presence of pure astigmatism of the cylindrical lens.

\subsection{Testing progressive lenses}

The RGi can be also applied to give demonstration about progressive focal length lenses used for eyeglasses. Using the same configution described in the section 3.4. Infact those lenses have a focal length that varies from the top to the bottom, to aid correct near and far vision. In Fig. 6 is shown as appear a fringe pattern when is imaged by a progressive lens. It should be noted that number fringes changes along the vertical direction giving information about the distribution of the focal power.

\section{REFERENCES}

1. Nachman P. "Mach-Zehnder interferometer as an istuctional tool", Am.J.Phys. 63 (1) (1995).

2. Chang F.P. and Reid G.T. Opt. Lasers Eng. 1277 (1990).

3. S. De Nicola, P.Ferraro, A.Finizio, G.Pesce and G.Pierattini, "Reflective Grating Interferometer for measuirng the refractive index of transparent materials", Opt.Comm., 118, 491 (1995).

4. M. de Angelis, S. De Nicola, P. Ferraro, A.Finizio and G. Pierattini, "A reflective grating interferometer for measuirng the refractive index of liquids", Pure Appl. Opt., 5, 761-765 (1996).

5. S. De Nicola, P. Ferraro, A.Finizio and G. Pierattini, "Refcletive Grating Interferometer for measuring the focal length of a lens by digital moiré effect", Opt.Comm., 132, 432 (1996).

6. M. de Angelis, S. De Nicola, P. Ferraro, A.Finizio and G. Pierattini, "An new apporach to high accuracy measurement of the focal lengths of lenses using a digital Fourier transform", Opt.Comm., 136, 370-374 (1997).

7. E.Keren, K.M.Kreske, and O.Kafri, "Universal method for determining the focal length of optical systems by moiré deflectometry" Appl.Opt., 27, 1383 (1988)

8. Y. Nakano and K. Murata, "Talbot Interferometry for measuring the focal length of a lens" Appl. Opt., 24, 3162 (1985).

9. D.Malacara, "Radial, Rotational, and Reversal Shear Interferometers", in Optical Shop Testing, D.Malacara, ed. (Wiley, New York, 1992, pp.173-206.

10. same of reference 9., p.78.

11. same of reference 9., p.329.

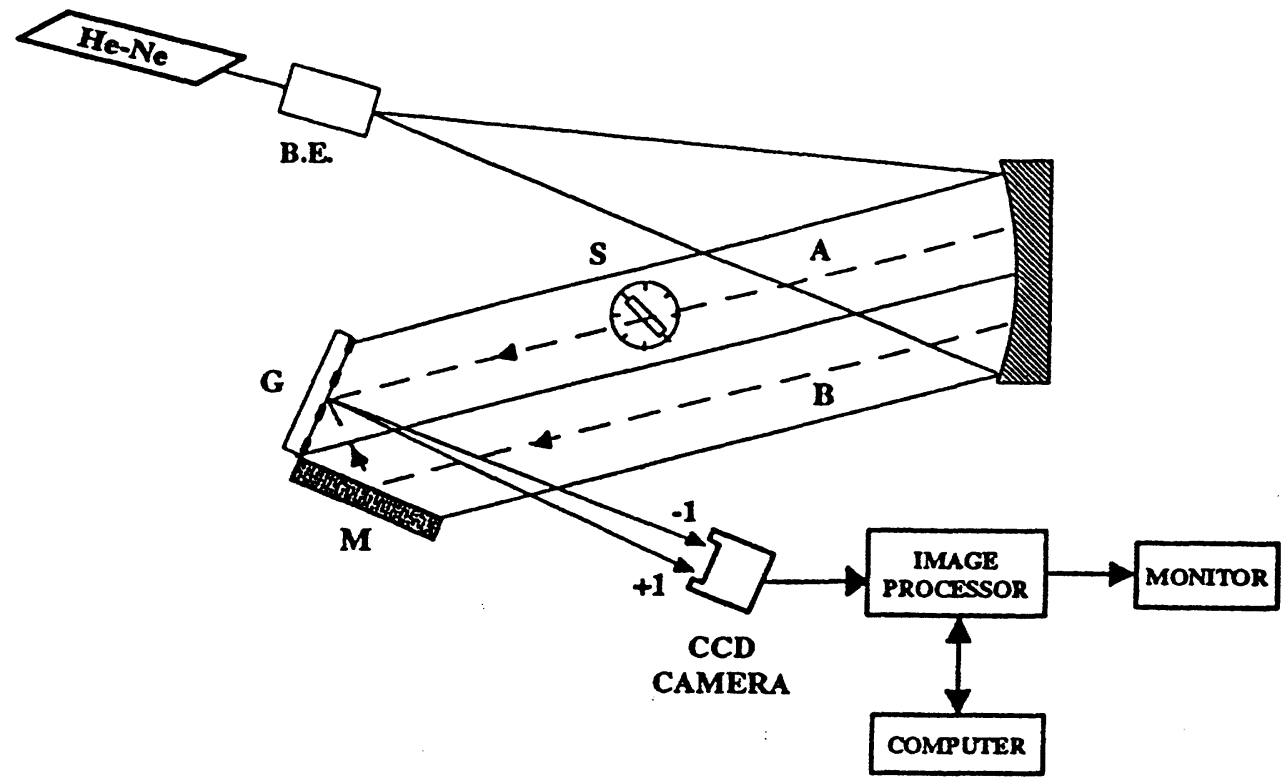

Fig.1 Optical set-up of the Reflective Grating Interferometer; M-mirror; G-diffraction grating. 


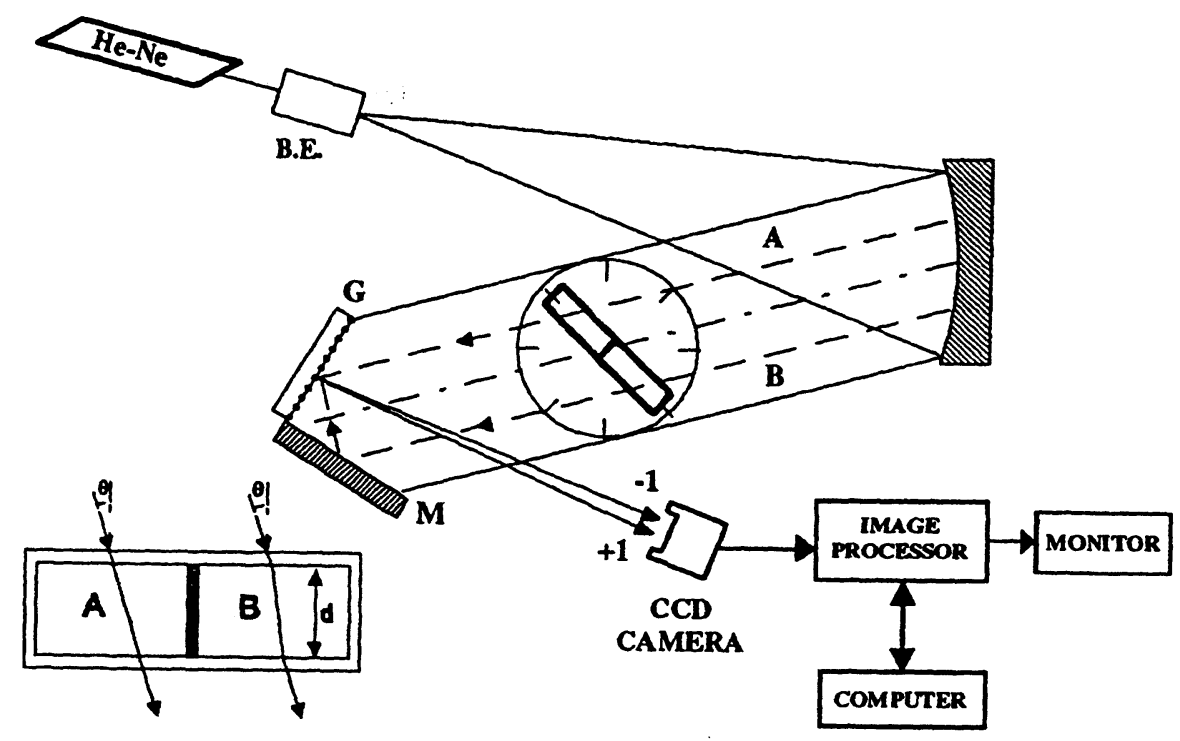

Fig.2 Optical configuration for measuring refractive index of liquids.

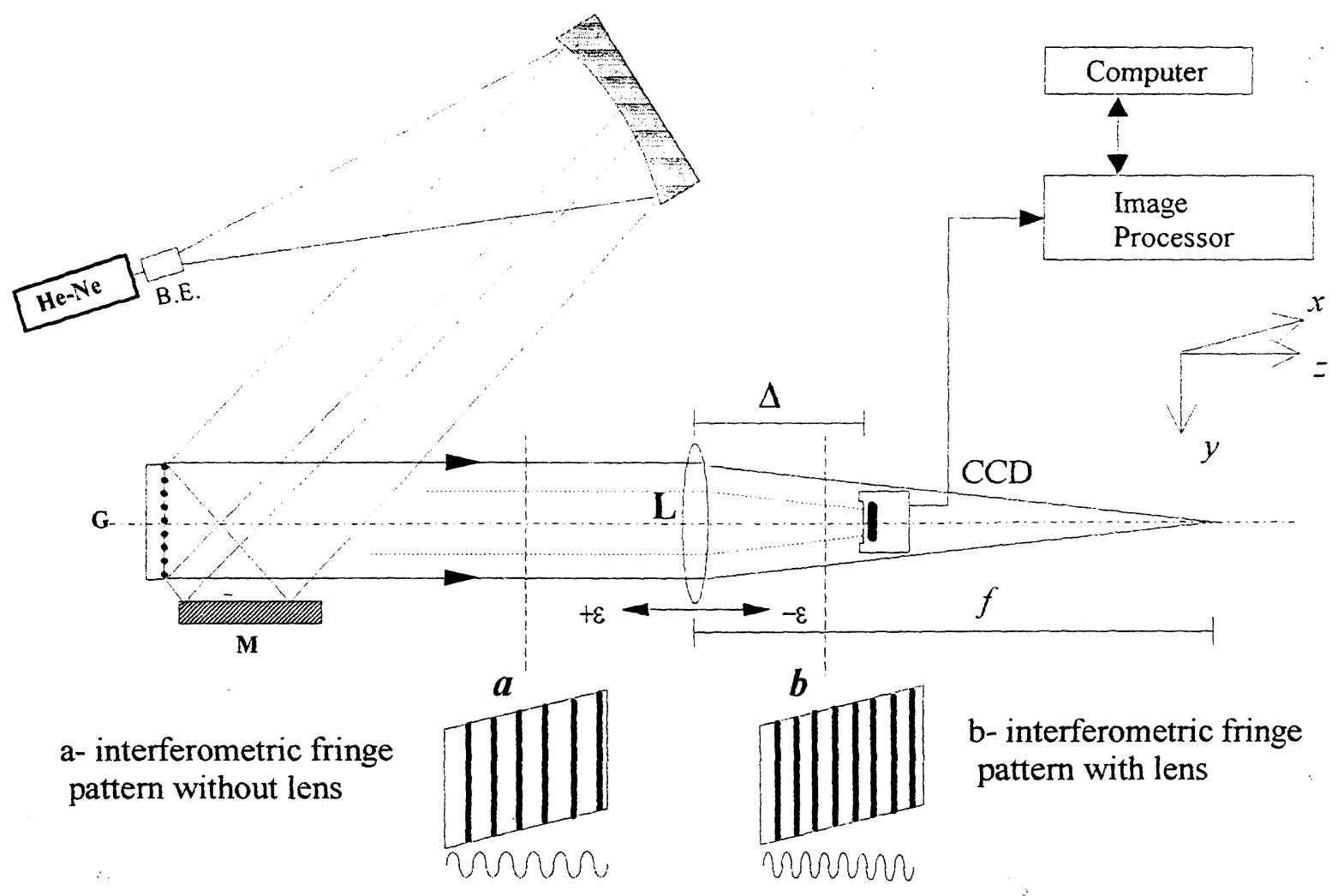

Fig.3 Optical for determinig focal length of a lens 


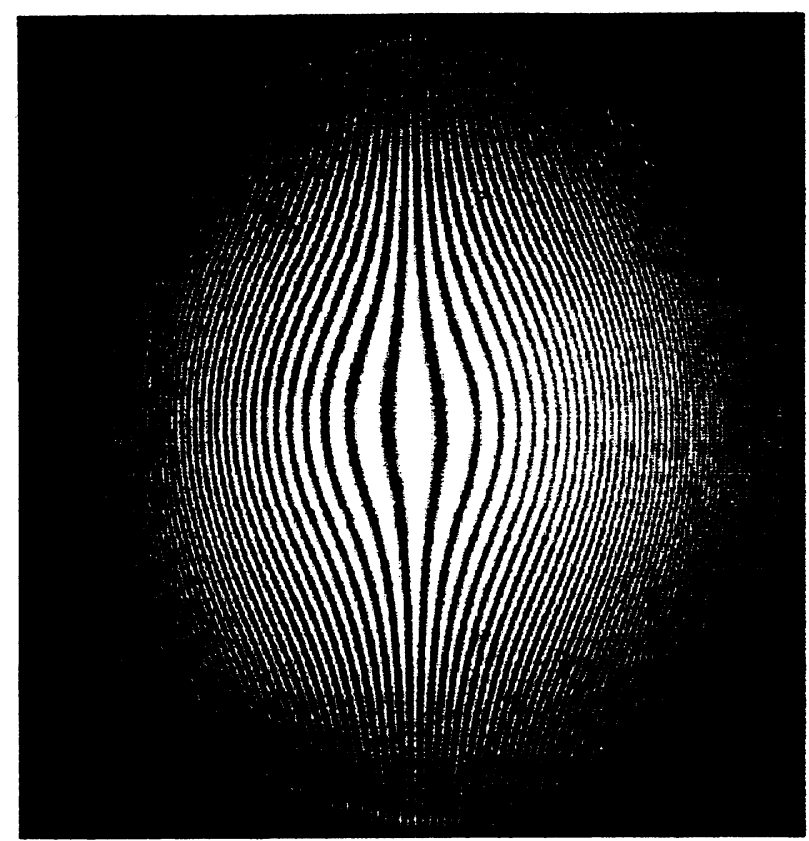

(a)

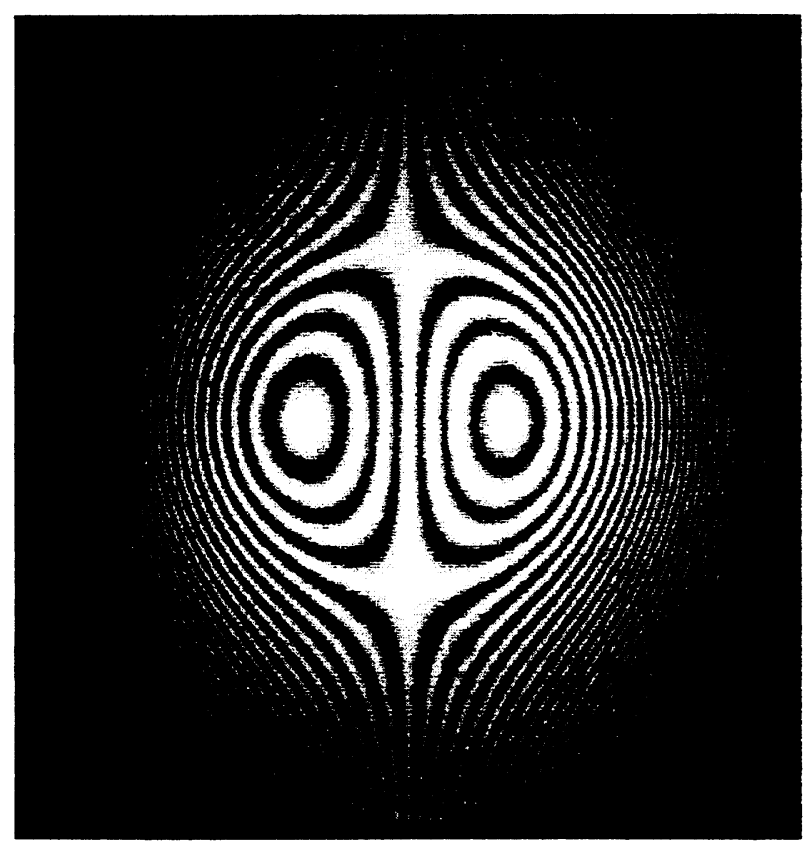

(c)

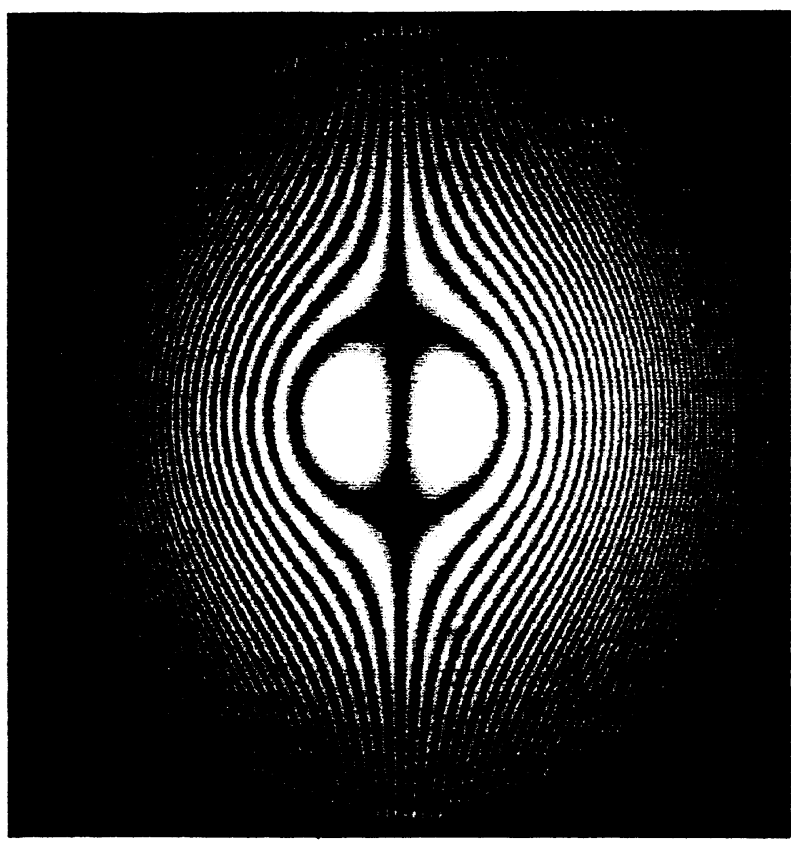

(b)

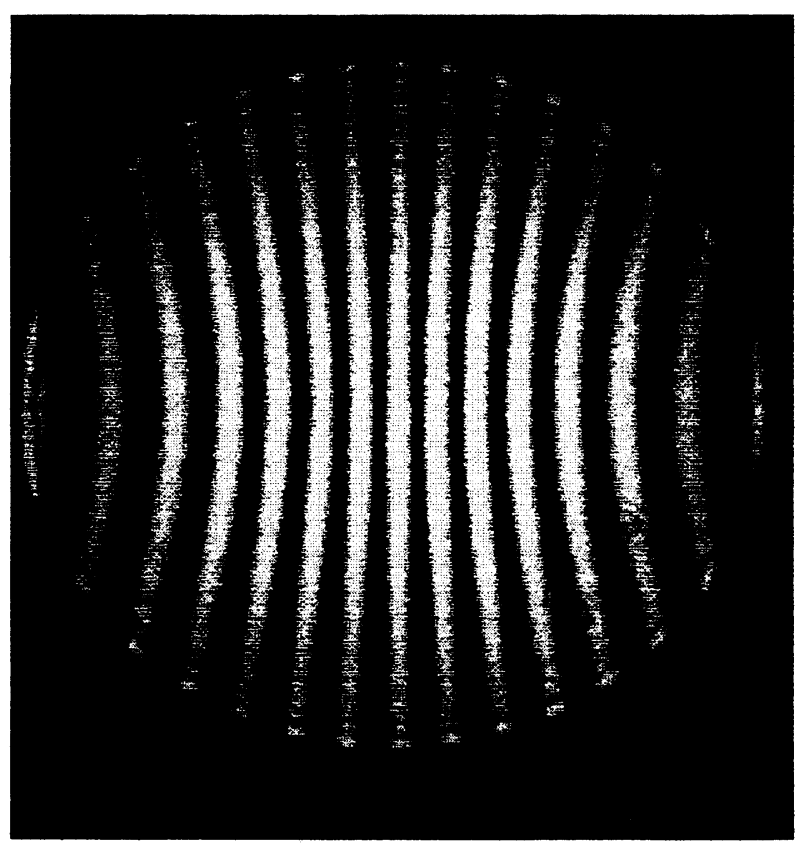

(d)

Fig.4 Aberration of a lens obtained using a Reflection Grating Interferferometer (see the text). 


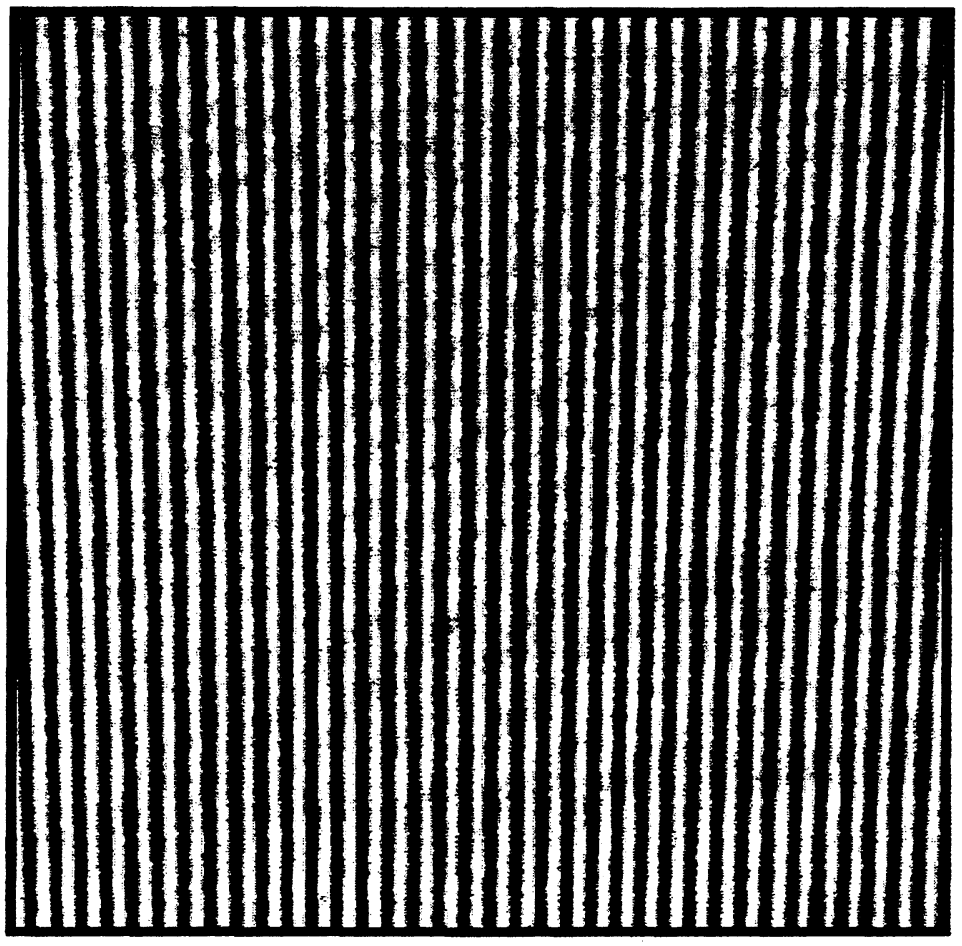

Fig. 5 A parallel fringe pattern when imaged by a progressive lens 